\title{
DESENVOLVIMENTO DE DESIGN MODULAR PARA ROV BIOINSPIRADO
}

Robson Costa Santiago (robsoncsantiago@gmail.com) - Centro de Engenharia, Modelagem e Ciências Sociais Aplicadas, Universidade Federal do ABC.

Magno Enrique Mendoza Meza (m.e.m.meza@gmail.com) - Centro de Engenharia, Modelagem e Ciências Sociais Aplicadas, Universidade Federal do ABC.

Silvia Lenyra Meirelles Campos Titotto (titotto@gmail.com) - Centro de Engenharia, Modelagem e Ciências Sociais Aplicadas, Universidade Federal do ABC.

\section{RESUMO}

ROV (Remotely Operated Vehicle) é um equipamento versátil e amplamente utilizado nas variadas operações das plataformas de petróleo, inclusive tendo aplicabilidade no campo militar. É um veículo submarino não tripulado, controlado pela superfície através de um cabo umbilical responsável pela alimentação e comunicação com o mesmo, e extremamente importante devido sua capacidade de atingir locais e profundidades praticamente inacessíveis para mergulhadores humanos, tornando-se prático e, principalmente, seguro. A proposta deste paper é apresentar uma revisão bibliográfica de temas correlacionados ao design e projeto de um ROV bioinspirado modular, que será concretizado através de um projeto de iniciação científica em andamento. Procura-se incorporar a eficiência animal no sistema, além de buscar o máximo de hibridismo possível no projeto, que vão desde, mas não se atendo somente a, braços robóticos, esteiras rolantes e propulsores, permitindo sua operação nos mais diversos ambientes. Será autônomo com possibilidade de funcionamento via baterias (portanto, configurando-se também como um AUV-Autonomous Underwater Vehicle, ou Veículo Submarino Autônomo), possuindo, porém, acesso a um cabo umbilical caso desejado. Esta característica amplia a gama de aplicações do equipamento, trazendo ainda mais versatilidade ao projeto. Como será possível operar tanto como um ROV, quanto como um AUV, denomina-se então como um HROV (Hybrid Remotely Operated Vehicle ou Veículo Híbrido Remotamente Operado).

Palavras chave: Remotely Operated Vehicle; Modular; Biomimética; Hibridismo; Autônomo. Área: $O$ design e o desenvolvimento de produtos e serviços 


\section{INTRODUÇÃO}

\subsection{Contextualização, recorte do problema e objetivos}

ROVs são veículos não tripulados controlados e monitorados pela superfície, possuindo múltiplas funções, atreladas principalmente às áreas petrolífera, militar, de exploração oceânica e inclusive para educação. Uma das aplicações iniciais dos ROVs foi na área militar: na década de 1950, a marinha da Grã-Bretanha utilizou um submersível (veículo pequeno submarinho) para recuperação de torpedos e remoção de minas navais (TAHIR e IQBAL, 2014).

Figura 1: ROV CUTLET - Marinha Britânica

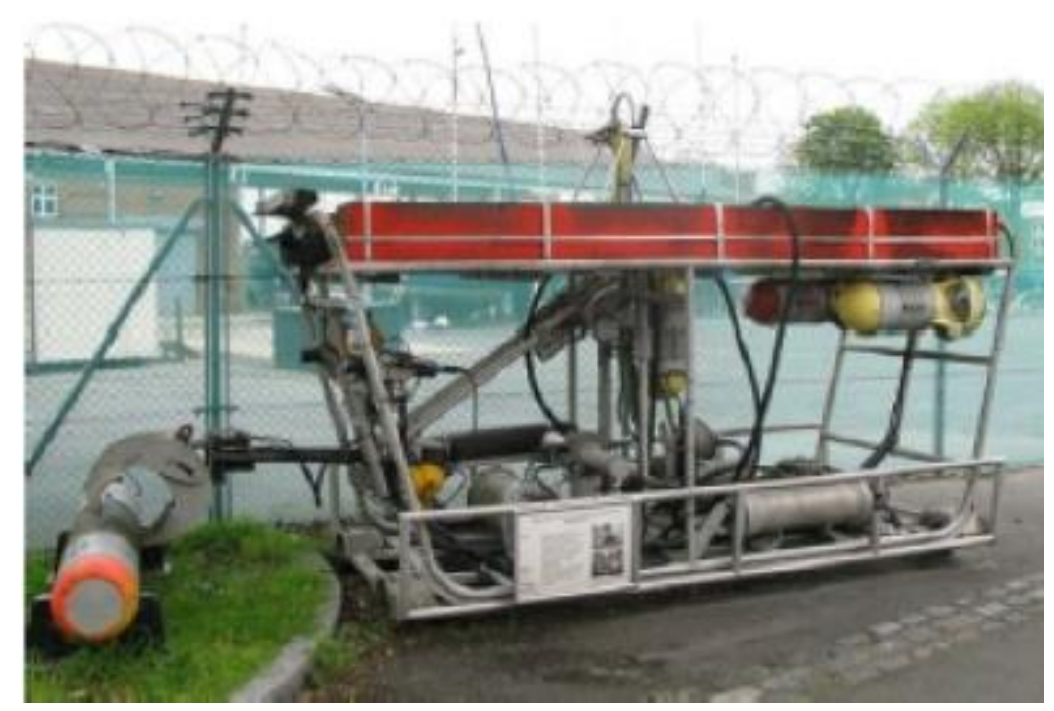

Fonte: TAHIR e IQBAL, 2014, p. 1112

Já na década de 60, os chamados CURVs (Cable-Controlled Underwater Recovery Vehicles Veículos de Recuperação Subaquática controlados por cabos) foram empregados em operações de resgate e recuperação em mar profundo pela marinha dos Estados Unidos. Evoluíram, então, para os CURV II e CURV III no início da década de 70 (TAHIR e IQBAL, 2014).

Com o desenvolvimento da indústria offshore nas décadas de 80 e 90, passou-se a empregar ROVs militares também nas áreas de óleo e gás, educação e pesquisa oceânica. Hoje, estes desempenham papéis importantíssimos e são amplamente utilizados na exploração oceânica ao redor do mundo e instalações/operações subaquáticas (TAHIR e IQBAL, 2014).

Se tratando de ROVs híbridos, é importante mencionar o Nereus, um HROV projetado pela WHOI (Woods Hole Oceanographic Institution). Conforme afirmou Fletcher e Yoerger - um dos pesquisadores mais importantes do ramo - as possibilidades provenientes da natureza híbrida deste sistema disponibiliza um método financeiramente eficiente para exploração e pesquisa no fundo do oceano. Grande parte das bacias oceânicas está a profundidades além da capacidade de robôs atualmente utilizados (FLETCHER et al., 2005). Este equipamento foi utilizado em estudos até maio de 2014, quando se perdeu comunicação com o mesmo (suspeita de implosão devido à alta pressão).

Atualmente os ROVs são empregados em diversas áreas. Sua demanda desperta o interesse de cientistas em buscar novas possibilidades de design, implementações de equipamentos e ferramentas e como torná-lo mais eficiente. 
Neste paper, é apresentada uma revisão bibliográfica dos temas ligados ao objeto de uma pesquisa em andamento, com o intuito de apresentar o estado da arte a respeito destas áreas, e resultados preliminares da iniciação científica em andamento, que se propõe a caracterizar um design bioinspirado (biomimética) de um ROV, buscando um tamanho compacto que seja capaz de abrigar os equipamentos destinados ao dispositivo, além de manter elevado grau de modularização, permitindo múltiplas configurações. Posteriormente, serão avaliados também a implementação e interfaceamento de sensores com o próprio design.

\subsection{Justificativa}

Buscar designs mais compactos e eficientes, maior autonomia e baixos custos são desafios e objetos de pesquisa atualmente. Tamanhos mais compactos implicam em facilidade de transporte, economia de materiais, possibilidade de inspeções em áreas de difícil acesso, menor peso e melhor eficiência hidrodinâmica/aerodinâmica, que reflete em melhor resposta de controle e menor consumo de energia, aumentando a autonomia, segundo ponto mencionado. Este implica em maior quantidade de dados coletados e menor custo de operação, visto que demanda menos retornos à embarcação ou à superfície (recarga por painéis solares), e maior alcance do equipamento. Por último, baixos custos seriam características importantíssimas no mercado atualmente, visto que o preço de um ROV comercial é extremamente alto, o que tornaria a tecnologia mais acessível inclusive para missões menores ou até mesmo objeto de aprendizado nas mais diversas áreas a que ele se aplica.

\section{BIOMIMÉTICA}

Este ramo é responsável pela análise da natureza e reprodução de seus fenômenos e características através de sistemas sintéticos (KATCHBORIAN, 2016). Sabe-se que seresvivos, por terem passado por milhões de anos de evolução, são sistemas altamente eficientes, onde a própria seleção natural esculpiu as características que apresentam os melhores resultados nos mais diversos ambientes. Desse modo, procura-se inspiração animal no design mecânico do ROV, buscando incorporar tais mecanismos de eficiência em um sistema robótico (MCKENNA, 2011).

Quando se fala de biomimética e de sua relevância, devemos levar em consideração publicações de autores importantes como Yoseph Bar-Cohen, que publicou diversos artigos referentes a esta área, além do livro "Biomimetics: Biologically Inspired Technologies". Neste, Bar-Cohen ressalta que enquanto o processo de evolução continua, a natureza continua a criar soluções eficazes que podem ser imitados ou servir de base para criação de tecnologias inovadoras. Dos numerosos exemplos de aplicação da biomimética, ele cita o exemplo do formato de "favo de mel", utilizada em estruturas aeroespaciais por proporcionar alta resistência e baixo peso, que, embora cientistas aleguem que o formato não foi copiado das abelhas, este já estava presente na natureza antes da chegada dos humanos, o que impede que seja concedida uma patente ao pesquisador que tenha estudado primeiro esta geometria (BAR-COHEN, 2006).

O design do ROV influencia nas características hidrodinâmicas do mesmo, portanto, a aplicação da biomimética pode colaborar na eficiência do veículo. Não só no design pode incorporar-se a biomimética, como, futuramente, nas partes sensoriais, de manobrabilidade e propulsão do mesmo. Estudos estão sendo conduzidos nestas áreas para buscar soluções bioinspiradas eficientes. Segundo McKenna (2011 apud BANDYOPADHYAY, 2009; ELLINGTON, 1984; DICKINSON et al., 1999), uma nova forma promissora de propulsão é o uso de propulsores de lâmina de hiper-sustentação bioinspirados. Produziu-se uma série de 
lâminas rígidas de hiper-sustentação, que se assemelhavam a nadadeiras de pinguins, e caracterizou-se suas propriedades de propulsão, sendo posteriormente utilizadas em um veículo submarino. Um novo propulsor chamado "Slosher" foi posteriormente desenvolvido, possuindo múltiplas lâminas dispostas radialmente, podendo operar tanto como propulsor de hiper-sustentação em baixas velocidades ou, com as lâminas travadas, como um propulsor padrão a altas velocidades. Isso evita a banda morta de controlabilidade em baixas velocidades.

Como exemplo de sensores bioinspirados, podemos citar bigodes artificiais que atuam simulando os bigodes encontrados em diversos roedores. Estes já foram implementados em robôs para testes e os guiam evitando colisões com obstáculos em seus caminhos. Outro exemplo são narizes eletrônicos, já comercialmente disponíveis, empregados atualmente em monitoramento ambiental e controle de qualidade em áreas como processamento de alimentos. Estes dispositivos são compostos por um conjunto de sensores químicos específicos, que simulam os narizes de mamíferos, por reconhecimento de padrões. Assim, gases e suas misturas podem ser identificadas pelo padrão das respostas obtidas pelos sensores aplicados no dispositivo (BAR-COHEN, 2006).

\section{ROBÓTICA BIOINSPIRADA}

Diversos estudos são realizados ao redor do mundo nesta área, e demonstram que os resultados de se imitar a natureza e agregar tais características aos sistemas robóticos se mostram cada vez mais promissores.

Um dos grandes nomes desta tendência, Robert Full, produziu diversos artigos sobre este tema - como "The Dynamics of Legged Locomotion: Models, Analysis and Challenges" (2006) - e apresentou ao TED algumas de suas aplicações, como a descoberta das estruturas responsáveis pela aderência de lagartixas às superfícies. Posteriormente, cientistas confeccionaram materiais sintéticos que fossem análogos às estruturas presentes nestes animais, permitindo a criação de adesivos com alta força de adesão e abriu caminho para estudos de robôs que se comportassem como as lagartixas, aderindo às superfícies imitando a técnica animal. Também se estudou a utilidade de suas caudas, simulando, em um robô, como estes animais são capazes de manobrar e controlar seu corpo em queda utilizando a cauda.

Dennis Hong afirma que conforme a tecnologia da robótica avança, novas aplicações surgem, trazendo consigo dificuldades e obstáculos aos padrões dos robôs atualmente. São necessárias alternativas de locomoção, por exemplo, que permitam acesso aos mais variados ambientes irregulares dependendo de suas aplicações - robôs de resgate onde atuam em meio a escombros. Hoje, basicamente, a movimentação de robôs se dá por rodas ou pernas, o que torna inviável ou extremamente difícil esta aplicação ao exemplo citado anteriormente. São motivações como estas que pesquisas são conduzidas a respeito de novas possibilidades de locomoção para robôs, e a natureza oferece modelos e processos que podem servir de inspiração para o desenvolvimento de novas tecnologias (HONG, 2006).

Podemos citar também a área correlacionada Soft Robotics, que busca encontrar maneiras de projetar e construir robôs que possuam poucas partes rígidas, ou mesmo nenhuma. São pesquisados materiais que sejam deformáveis, ou estruturas que permitam ao robô uma melhor interação com ambientes dinâmicos e irregulares. Estes dispositivos poderiam, por exemplo, interagir fisicamente com substâncias frágeis ou se deformar para transpassar uma fenda. Hod Lipson pontua que embora os avanços na ciência dos materiais estejam trazendo materiais macios e robôs flexíveis mais perto da realidade, ainda existem desafios que permeiam as áreas de design e controle. Por exemplo, um sistema que tende a ser o mais 
deformável possível, tende a possuir menos espaço e potência para os atuadores. Então, como controlar uma estrutura que contém mínima quantidade de partes rígidas? Esta é a pergunta que guia os estudos e pesquisas nesta área (LIPSON et al., 2008).

Figura 2: Robô inspirado na lagarta da manduca. Exemplo de aplicação do soft robotics. Fonte: Rieffel $\boldsymbol{e t}$ al. (2008)

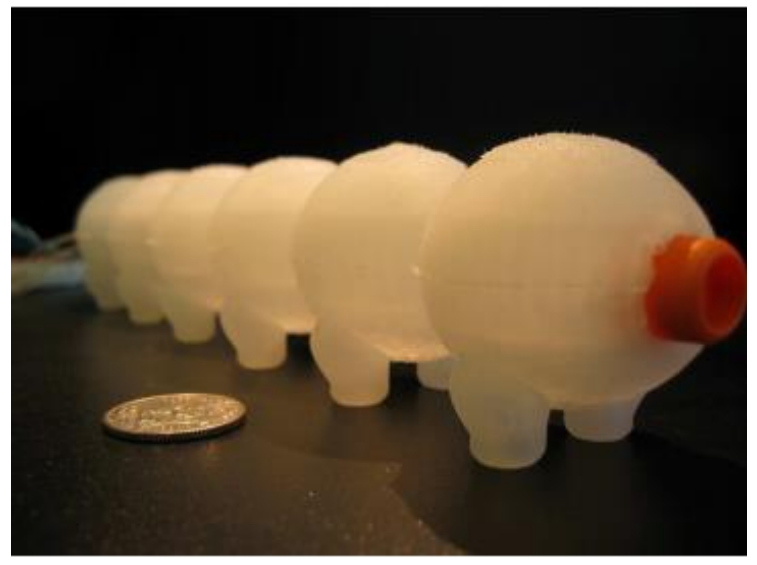

Abaixo, apresenta-se um conceito bioinspirado inovador, um robô chamado de PoseiDRONE, inspirado nos polvos. Este é um protótipo construído em pequena escala para demonstrar as ideias por trás deste design, onde Arienti e seus colegas (2013) afirmam que sua estrutura é praticamente toda constituída de materiais macios (soft materials) e capaz de unir três funções diferentes: locomoção por rastejamento, nado e possibilidade de manipulação de objetos. Além disso, a aplicação destes materiais permite que o robô possa se compactar de modo a passar por pequenas aberturas, e diminui seu peso, facilitando seu transporte. Sua capacidade de deformação também auxilia na resistência à impactos, fornecendo mais durabilidade ao robô.

Figura 3: PoseiDRONE, robô bioinspirado em polvos, procurando unir habilidades de rastejamento e nado inspirados no animal. Fonte: Arienti et al. (2013)
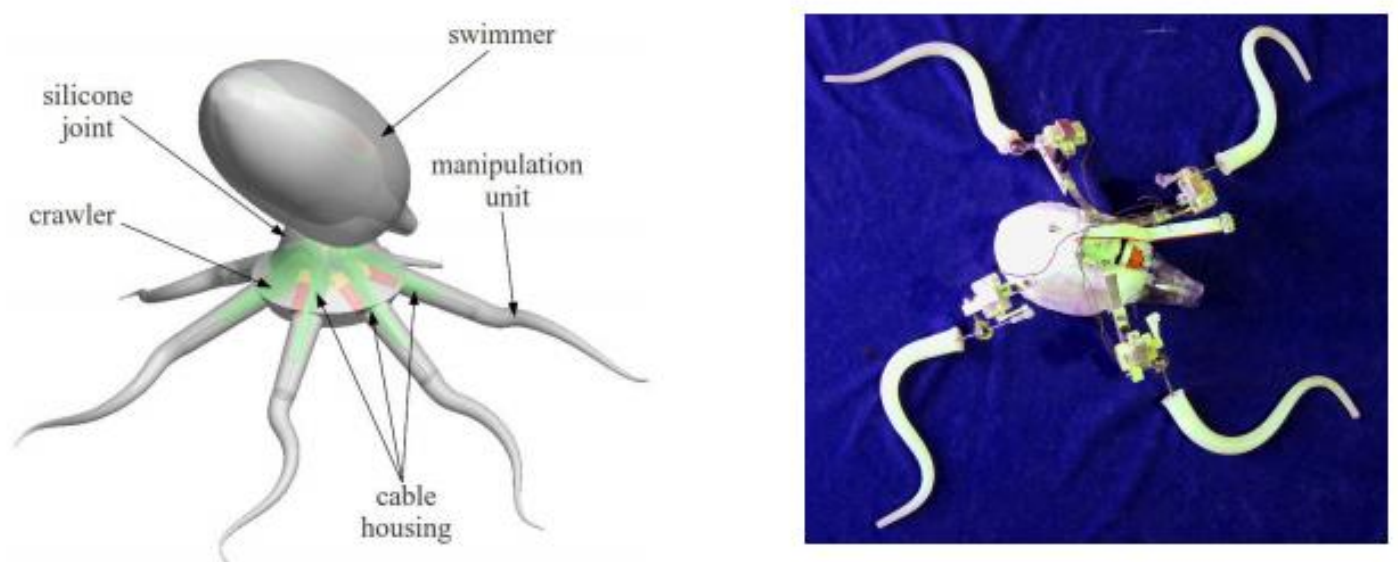

\section{DESAFIOS E LIMITAÇÕES}

Uma característica importante dos ROVs atuais que precisa ser ressaltada é o seu preço. 
Segundo um estudo realizado pela IFR (International Federation of Robotics - Federação Internacional de Robótica) em 2010, o preço de um sistema submarino (compreendendo ROVs e AUVs - Veículos Submarinos Autônomos) girava em torno de 850 mil dólares, tendo o sétimo maior preço dentre diversos tipos de robôs (TAHIR e IQBAL, 2014). Portanto, conciliar o projeto de um ROV a um preço mais baixo é outro desafio a ser vencido, o que traria grande visibilidade ao projeto.

Alta capacidade de modularização também será um desafio a ser superado, aliando variabilidade a um design compacto observadas as características originais do projeto. Um módulo de baterias está previsto para que o veículo possa se comportar como um AUV, o que traz uma vasta gama de aplicabilidades, como estudos de vulcanismo submarino e fontes hidrotermais, mapeamento e monitoramento de escape de fluidos de baixa temperatura e de ecossistemas quimiossintéticos, mapeamento de habitats bentônicos (próximos do fundo oceânico), e mapeamento de características morfológicas do solo oceânico (WYNN et al., 2014), aplicações estas na área de geologia marítima, o que demonstra a alta variabilidade de usos desta tecnologia.

Estudos mostram que tendências futuras no estudo de robótica submarina apontam para obtenção de tecnologias que sejam altamente eficientes energeticamente, com autonomia de longa duração que possibilitariam maior alcance das missões dos AUVs. Também são buscadas soluções que sejam capazes de "coletar" energia nos ambientes marítimos, partindo de processos situados in situ. Quanto à robótica bioinspirada, Zhang e seus colegas (2015) afirmam que um dos grandes desafios atualmente é de procurar formas de melhorar a densidade e eficiência energética de propulsão de modo que estas tecnologias inovadoras possam se equiparar e competirem com as tecnologias tradicionais. Muitos destes equipamentos são apenas protótipos e testados em laboratório ou locais naturais moderados. Porém, espera-se que estas tecnologias sejam postas em prática apresentando alternativas aos métodos atuais.

Já Bandyopadhyay (2016) pontua que o grande potencial desta área ainda é limitado pela falta de avanços nas tecnologias de músculos artificiais, além de dificuldades na parte de controle temporal não-linear e integração entre este e a parte de sensoriamento. Mas acredita-se que a partir do momento que estas tecnologias avançarem, ótimos resultados surgirão na precisão naval e propulsão de veículos submarinos, principalmente.

\section{RESULTADOS ESPERADOS}

Este paper apresenta considerações iniciais do projeto de iniciação científica em andamento e resultados obtidos pela análise da bibliografia aqui apresentada. Posteriormente, será feita uma revisão da bibliografia complementar procurando incorporar aspectos importantes ao desenvolvimento do design. Espera-se ainda obter o aspecto da carcaça do ROV, juntamente com o material que comporá a mesma, e então realizar testes em escala reduzida utilizando uma impressora 3D para composição do protótipo inicial. Paralelamente ao levantamento do design do ROV, serão estudados e caracterizados os sensores comumente utilizados nestes robôs, como velocímetro Doppler, GPS, profundímetro e altímetro, etc. Serão analisadas suas características, também como objeto de aprendizagem ao longo da iniciação científica, porém atentando-se a características que sejam relevantes ao design do ROV.

\section{CONCLUSÃO}

A área de robótica vem sendo objeto de intensa pesquisa atualmente e se inova cada vez mais ao passar do tempo. Os veículos submarinos tendem a se beneficiar deste progresso 
tecnológico e o faz de maneira recíproca: avanços nesta área trazem inovações em áreas adjacentes e vice-versa. Buscar alternativas que reduzam o preço de um ROV, além de prolongar a autonomia dos AUVs e aperfeiçoar a operabilidade destes equipamentos são objetivos comuns encontrados em pesquisas ao redor do mundo. O presente paper buscou sintetizar os temas que permeiam este projeto de pesquisa de maneira a apresentar um panorama sobre como estas áreas evoluíram e estão evoluindo atualmente. Estão sendo investidos esforços na concepção de um design que procure convergir vantagens destes temas e que permita otimizar o funcionamento do robô: modularidade e hibridismo visando versatilidade com olhares na biomimética.

\section{REFERÊNCIAS}

ARIENTI, A.; CALISTI, M.; GIORGIO-SERCHI, F.; LASCHI, C. PoseiDRONE: design of a soft-bodied ROV with crawling, swimming and manipulation ability". Procedimentos da conferência MTS/IEEE OCEANS, 2013. San Diego, CA, Estados Unidos.

BANDYOPADHYAY, P. R.; Highly Maneuverable Biorobotic Underwater Vehicles. In: DHANAK, M. R.; XIROS, N. I. (Eds.). Springer Handbook of Ocean Engineering. Nova lorque: Springer International Publishing, 2016. p. 281-300.

BAR-COHEN, Y. Introduction to Biomimetics: The Wealth of Inventions in Nature as an Inspiration for Human Innovation. In:

Estados Unidos: CRC Press, 2006. P. 1-40. (Ed.). Biomimetics: Nature-Based Innovation,

FLETCHER, B. et al. The Hybrid Remotely Operated Vehicle (HROV): New Challenges and Opportunities. Disponível em:<

http://www2.hawaii.edu/ bsb/docs/HROV for UI 2005 Dec 02 bfletcher compressed.pdf> . Data de acesso: 17/05/2017.

HONG, D. W. Biologically Inspired Locomotion Strategies: Novel Ground Mobile Robots at RoMeLa, 3rd International Conference on Ubiquitous Robots and Ambient Intelligence (URAI), 2006. Seul, Coréia do Sul.

KATCHBORIAN, Pedro - Conheça a biomimética, a arte que imita a natureza. Disponível em: $<$ https://www.freetheessence.com.br/sustentabilidade/negocios/biomimetica-ciencianatureza/>. Acesso em 05/05/2017.

MCKENNA, Thomas. Developing Bioinspired Autonomous Systems. Marine Technology Society Journal, Maryland, v. 45, n. 4, p. 19-23, jul./ago. 2011.

RIEFFEL, J.; TRIMMER, B.; LIPSON, H. Mechanism as Mind: What Tensegrities and Caterpillars Can Teach Us about Soft Robotics. Disponível em: $<$ http://cs.union.edu/ rieffelj/papers/rieffel-alifexi-2008.pdf>. Data de acesso: 02/06/2017.

TAHIR, A.; IQBAL, J. Underwater Robotic Vehicles: Latest Development Trends and Potential Challenges. Science International, Lahore, v. 26, n. 3, jul./ago. 2014. Disponível em: <http://www.sci-int.com/pdf/7039375991111-1117--AHMAD\%20MAMOOD\%20TAHIR-NUST--IT-FINAL.pdf> Data de acesso: 10/05/2017.

WYNN, R. B. et al. Autonomous Underwater Vehicles (AUVs): Their past, present and future contributions to the advancement of marine geoscience. Marine Geology, Reino Unido, v. 352, p. 451-468, jun 2014. Disponível em: <http://www.sciencedirect.com/science/article/pii/S0025322714000747> Data de aceso: 11/05/2017.

ZHANG, F.; MARANI, G.; SMITH R. N.; CHOI, H. T. Future Trends in Marine Robotics. IEEE Robotics and Automation Magazine, Nova lorque, v. 22, n. 1, mar. 2015. Disponível em: <http://ieeexplore.ieee.org/document/7059360/>. Data de acesso: 02/06/2017. 